\title{
Photoemission Study of the Initial Growth of Co on $\mathrm{Ru}(0001)$
}

\author{
P.J. Godowski ${ }^{a, *}$, J. OnsGaARD ${ }^{b}$, \\ M. Trzebiatowska-GusowskA ${ }^{c}, \mathrm{~K}$. Pater $^{d}$ \\ AND ZHe SHEN LI ${ }^{e}$ \\ ${ }^{a}$ Institute of Experimental Physics, University of Wrocław \\ pl. Maxa Borna 9, 50-204 Wrocław, Poland \\ ${ }^{b}$ Department of Physics and Nanotechnology \\ Aalborg University, 9220 Aalborg East, Denmark \\ ${ }^{c}$ Institute of Low Temperature and Structure Research \\ P.A.S., P Nr 1410, 50-950 Wrocław 2, Poland \\ ${ }^{d}$ Institute of Physics, University of Technology \\ Wybrzeże Wyspiańskiego 27, 50-370 Wrocław, Poland \\ ${ }^{e}$ Institute of Storage Ring Facilities, University of Aarhus \\ 8000 Aarhus, Denmark
}

(Received February 6, 2007)

\begin{abstract}
The growth process of cobalt on $\mathrm{Ru}(0001)$ was characterized by photoelectron spectroscopy excited by X-ray and synchrotron radiation. The binding energy position and intensity of the Co $2 p_{3 / 2}$ and $\mathrm{Ru} 3 d_{5 / 2}$ core levels as well as the shape and structure of the valence band spectra corresponding to the different stages of the deposition were investigated. An observed small positive binding energy shift is a consequence of an increase in the cobalt adatoms coordination number. The core-level shift between bulk and surface Ru atoms is determined as $-360 \mathrm{meV}$. Upon adsorption of cobalt, the interface peak appears with a shift of $-(70 \div 80) \mathrm{meV}$ relative to the bulk one. On the basis of unchanged energy positions and widths of the $\mathrm{Ru}$-derived features of the valence band spectra, a weak interaction between cobalt and substrate is suggested. The measured valence band could be reproduced by superimposing the spectra of the pure elements.
\end{abstract}

PACS numbers: 79.60.Dp, 79.60.Jv, 68.55.Jk

*corresponding author; e-mail: pjg@ifd.uni.wroc.pl 


\section{Introduction}

Understanding of adsorbate-adsorbate and adsorbate-substrate interactions between two metals is an important issue for a variety of technological applications. The study of cobalt deposition on ruthenium is motivated by interest in thin magnetic film grown on a ruthenium substrate [1-3]. Taking into account that the lattice constant of $\mathrm{Co}$ and $\mathrm{Ru}$ is equal to 0.2506 and $0.2650 \mathrm{~nm}$, respectively [4], the misfit, $m$, is calculated as $m=-5.4 \%$ for the $\mathrm{Co} / \mathrm{Ru}$ interface. The phase diagram presented by Daruka and Barabási [5] for this $m$ predicts that at least up to two layers, the adsorbate should grow in a monolayer-by-monolayer (Frankvan der Merwe (FM)) mode. However, experimentally it was found [6] that the growth of cobalt follows a Stranski-Krastanov mode, i.e. after completion of a monolayer, three-dimensional (3D) islands start to appear on the substrate. More recently, El Gabaly et al. [7] reported a perfect FM growth mode at the substrate temperature of $460 \mathrm{~K}$. Despite of the above discrepancies, there is no doubt that in the submonolayer regime, cobalt deposited onto the $\mathrm{Ru}(0001)$ substrate at around room temperature (RT) forms monoatomically high islands of triangular shape. The first cobalt layer orders pseudomorphically on the ruthenium substrate.

To provide a better understanding of this system and obtain well-defined starting conditions for preparation of $2 \mathrm{D}$ alloys on the (assumed inert) Ru substrate, we carry out an experimental study of Co deposition on the Ru(0001) substrate. Photoemission spectroscopy using X-ray (XPS) $\left(\operatorname{Mg} K_{\alpha}\right)$ or synchrotron radiation (SR-PS) was employed for characterizing the system and obtaining information about the electronic structure of the submonolayer Co deposit. The revaluation of the $\mathrm{Co} / \mathrm{Ru}$ interface was made from the point of view of the shape and binding energy position of photoemission features as well as their connection to the adlayer morphology.

\section{Experimental}

The measurements were carried out on the beamline SGM1 at the ASTRID storage ring, ISA, Aarhus, Denmark [8]. The photons were incident at an angle of $40^{\circ}$ with respect to the surface normal and photoelectrons were collected by a $200 \mathrm{~mm}$ mean radius hemispherical electron energy analyzer (Scienta SES-200 type) along the surface normal. The Co $2 p$ level was excited by $\mathrm{Mg} K_{\alpha}$ X-rays. The total instrumental resolution is estimated as 650,185 , and $82 \mathrm{meV}$ for the Co $2 p_{3 / 2}, \mathrm{Ru} 3 d_{5 / 2}$, and valence band (VB) spectrum, respectively (Table). All electron energies reported here, binding energies (BEs), are given with respect to the Fermi level which is set to $0 \mathrm{eV}$. The uncertainty associated with the BE determination was made equal to the Fermi energy precision $(1253.600 \pm 0.012 \mathrm{eV})$ obtained using $\mathrm{Mg} K_{\alpha}$ radiation. It represents the stability and reproducibility of the detecting system and substantially exceeds the error originating from the line shape fit. 
TABLE

Instrumental parameters of the SGM1 beamline. The electron energy analyzer acceptance angle was $\Delta \alpha= \pm 9^{\circ}$. Total instrumental resolution is calculated as $\sqrt{\left(\Delta E_{\mathrm{ph}}\right)^{2}+\left(\Delta E_{\text {eea }}\right)^{2}}$.

\begin{tabular}{|c|c|c|c|c|c|c|c|}
\hline \multirow{3}{*}{ Spectrum } & \multicolumn{6}{|c|}{ Parameters } & \multirow{3}{*}{$\begin{array}{r}\text { Total } \\
\Delta E_{\text {instr }} \\
{[\mathrm{meV}]}\end{array}$} \\
\hline & \multicolumn{3}{|c|}{ photon/monochromator } & \multicolumn{3}{|c|}{ electron energy analyzer } & \\
\hline & $\begin{array}{c}h \nu \\
{[\mathrm{eV}]}\end{array}$ & $\begin{array}{c}\text { slit } \\
{[\mu \mathrm{m}]}\end{array}$ & $\begin{array}{l}\Delta E_{\mathrm{ph}} \\
{[\mathrm{meV}]}\end{array}$ & $\begin{array}{c}\text { pass en. } \\
{[\mathrm{eV}]}\end{array}$ & $\begin{array}{c}\text { slit } \\
{[\mathrm{mm}]}\end{array}$ & $\begin{array}{l}\Delta E_{\text {eea }} \\
{[\mathrm{meV}]}\end{array}$ & \\
\hline Co $2 p$ & 1253.53 & $x$ & 630 & 40 & 0.8 & 80 & 650 \\
\hline $\mathrm{Ru} 3 d$ & 350 & 50 & 166 & 40 & 0.8 & 80 & 185 \\
\hline VB & 40 & 50 & 13 & 40 & 0.8 & 80 & 82 \\
\hline
\end{tabular}

The $\mathrm{Ru}(0001)$ sample was mounted on a manipulator capable of conductive heating up to $1300 \mathrm{~K}$. Temperature measurements were accomplished by a $\mathrm{W}_{95} \mathrm{Re}_{5} / \mathrm{W}_{74} \mathrm{Re}_{26}$ (C-type) thermocouple, attached to the edge of the sample. The typical cleaning procedure involved successive cycles of $\mathrm{Ar}^{+}$ions bombardment $(1 \mathrm{keV}, 6 \mu \mathrm{A}, 20 \mathrm{~min})$ and annealing at $1300 \mathrm{~K}$. Surface cleanliness was verified by photoelectron spectroscopy. Before each measurement the sample was flashed to around $500 \mathrm{~K}$ to remove any residual carbon monoxide adsorbed on the surface.

Cobalt was deposited using the mini e-beam evaporator by the use of electron beam induced heating of the target material (four pockets, direct water cooling: model EGCO, Oxford Applied Research). The positive ion current, arising from ionization of the target vapour, was used for monitoring and stabilization of the evaporation rate. Evaporation of cobalt rod of $2 \mathrm{~mm}$ in diameter was accomplished by the use of a flux current of $9.8 \mathrm{nA}$ (4.04 A filament current; $2 \mathrm{kV}$ accelerating voltage; $10.5 \mathrm{~mA}$ emission current) which corresponds to the evaporation rate of 0.158 monolayers of cobalt on $\mathrm{Ru}(0001)$, AMLs/min (AML = adsorbate monolayer). The measured source ion current (flux) was preliminarily calibrated with the readings of a quartz crystal monitor. All depositions were performed and analyzed at $350 \mathrm{~K}$.

\section{Results and discussion}

\subsection{Co $2 p$ transition}

The experimental information concerning the Co $2 p_{3 / 2}$ spectrum (XPS) obtained during growth of the layer is collected in Fig. 1. The insets in Fig. 1 show the family of the spectra (top) and a typical example of the fitting procedure (bottom). The following values were obtained: Lorentzian width of $0.88-0.92 \mathrm{eV}$, singularity index, $\alpha$, of $0.246-0.256$, and Gaussian width of $0.35 \mathrm{eV}$. The Co $2 p_{3 / 2}$ peak intensity (circles) as a function of deposition time is approximated by a broken line with the first break at $370 \mathrm{~s}$ of evaporation indicating completion of a 


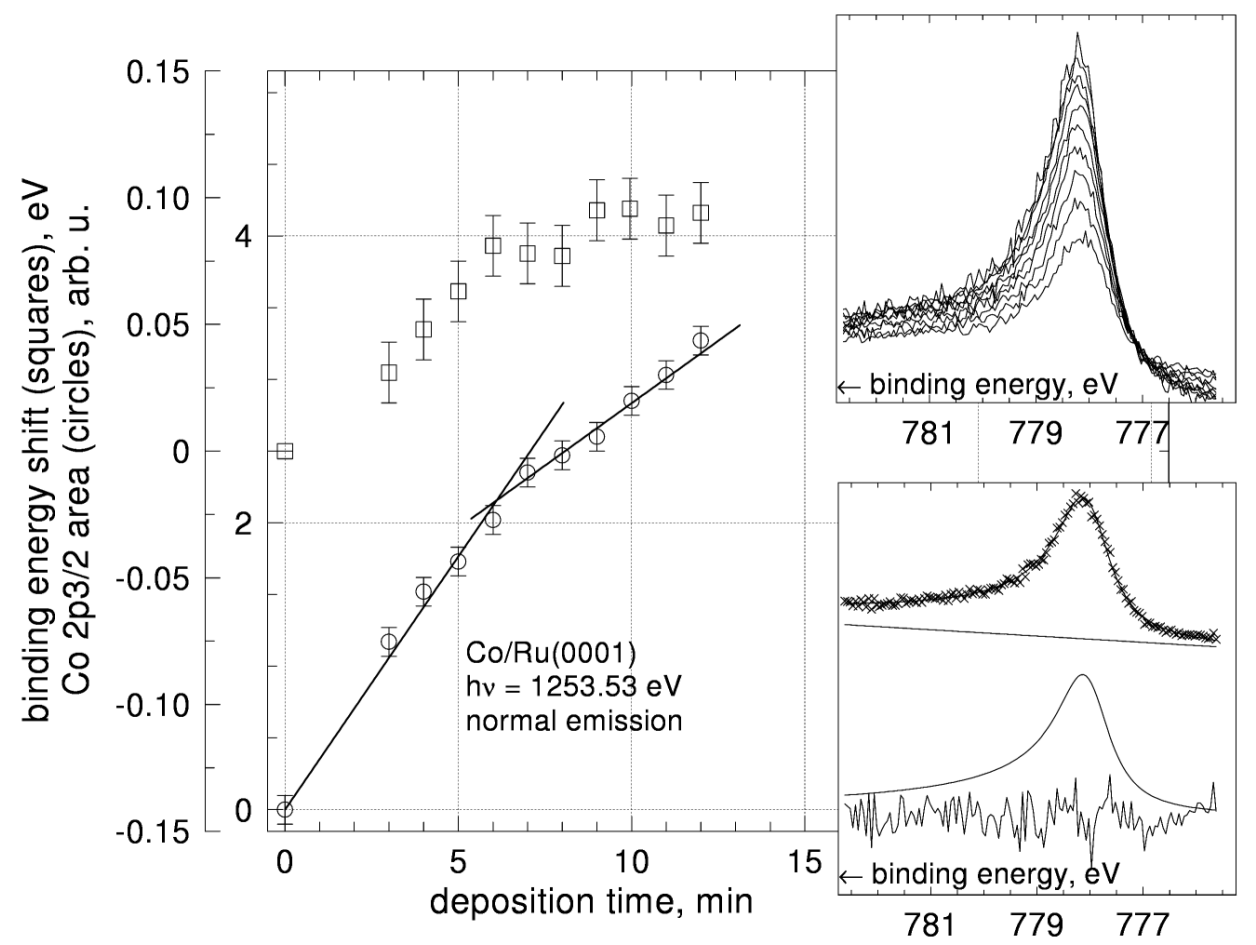

Fig. 1. Co $2 p_{3 / 2}$ photoelectron (XPS) intensity (circles) and binding energy (squares) as a function of cobalt deposition time on the $\mathrm{Ru}(0001)$ substrate. The insets show a collection of the spectra (top) and the typical result of fitting to the peak of $\theta=$ 0.62 AML (bottom) with residuals magnified by a factor of 5 for clarity.

monolayer. Within the cobalt peak shape, no indications which reflect the appearance of the second layer were found. As the zero point of $\mathrm{BE}$, the value of $778.000 \mathrm{eV}$ was assumed. The Co $2 p_{3 / 2}$ (squares) shifts slightly towards higher $\mathrm{BE}$ at the beginning of deposition and then after the intensity break-point becomes almost constant. From the first experimental point, corresponding to $c a$. 0.46 AML to 1 AML the peak shifts by $+0.062 \mathrm{eV}$. Calculated with the value of extrapolated data to zero coverage, the $\mathrm{BE}$ shift could be $+0.094 \mathrm{eV}$. It is worth mentioning that the chemical shifts for various Co compounds could be as large as $5.00 \mathrm{eV}[9]$ and the surface core-level shift (SCLS) of polycrystalline cobalt was determined as $-1.10 \mathrm{eV}$ [10]. The results in Fig. 1 do not show large changes in the electronic properties of $\mathrm{Co}$ monolayer bonded to $\mathrm{Ru}(0001)$. It is suggested that the gain in core-level $\mathrm{BE}$ is mainly a consequence of an increase in the coordination number of cobalt adatoms. Such situation takes place during the formation of $2 \mathrm{D}$ islands in contrary to the adsorbate distributed uniformly. In reality, deposition of cobalt on $\mathrm{Ru}(0001)$ at $\mathrm{RT}$ leads to the growth of triangular-shaped 2D islands [2]. 


\section{2. $R$ u $3 d_{5 / 2}$ transition}

Figure 2 shows the results of the substrate $(\mathrm{Ru})$ peak change during adsorption of cobalt as measured with SR-PS. The intensity of the $\mathrm{Ru} 3 d_{5 / 2}$ transition, determined as the area under the whole feature, decreases with the time of deposition. The change is approximated by two segments of the straight line with the breaking point at $390 \mathrm{~s}$ of evaporation. Together with the point of the intensity plot of the Co $2 p_{3 / 2}$ the time of $380 \mathrm{~s}$ was used for calibration of cobalt coverage as corresponding to $1 \mathrm{AML}$. The raw spectra of $\mathrm{Ru}$ are collected in the left inset starting from the clean surface (top spectrum). Typical results of analysis applied to the spectra are shown in the next insets (top and bottom right), which represent the clean $\mathrm{Ru}$ and $\mathrm{Ru}$ covered with $0.46 \mathrm{AML}$ of Co (as determined from the intensity changes), respectively. The Ru $3 d_{5 / 2}$ spectrum of the clean surface consists of clearly resolved two features at 279.745 and $280.105 \mathrm{eV}$ which are identified as originating from the surface $(\mathrm{S})$ and bulk (B) Ru atoms, respectively. Both compo-

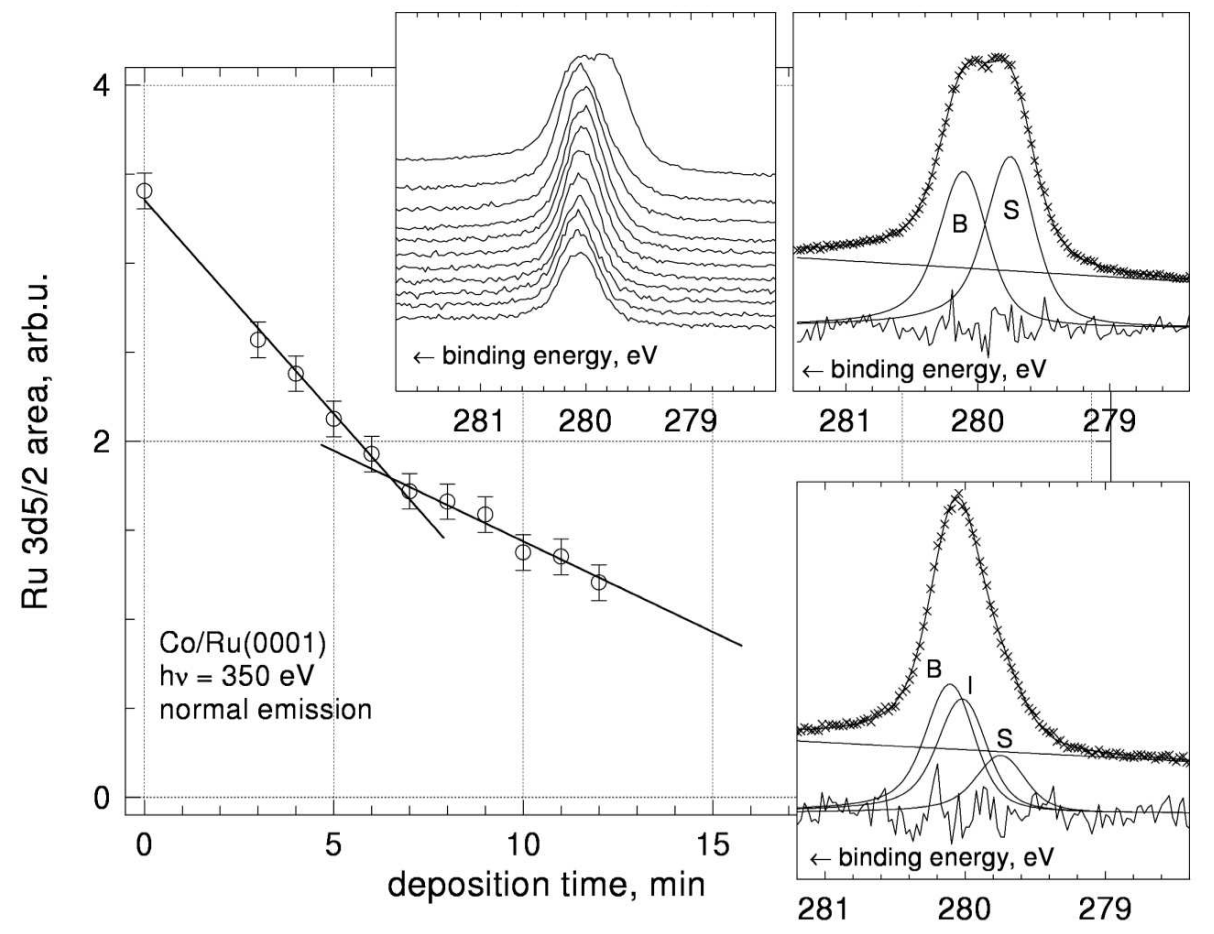

Fig. 2. $\mathrm{Ru} 3 d_{5 / 2}$ photoelectron (SR-PS) intensity as a function of cobalt deposition time on the $\mathrm{Ru}(0001)$ substrate. The insets show a collection of the spectra (top-left), the result of fitting to the peak of the clean substrate (top-right) and to the peak of $\theta=0.46$ AML (bottom) with residuals magnified by a factor of 5 for clarity. B denotes the contribution from the bulk of the sample, i.e. $\mathrm{Ru}_{\mathrm{Ru}}^{\mathrm{Ru}}$, $\mathrm{S}$ denotes from the surface, i.e. $\mathrm{Ru}_{\mathrm{Ru}}^{\mathrm{V}}$, and I - from the interface, i.e. $\mathrm{Ru} \mathrm{Ru}_{\mathrm{Ru}}^{\mathrm{Co}}$. 
nents are characterized by a Lorentzian width of $0.17-0.21 \mathrm{eV}, \alpha=0.053-0.058$, and a Gaussian width of $0.30 \mathrm{eV}$. The high resolution $\mathrm{Ru} 3 d_{5 / 2}$ spectrum acquired with a better experimental resolution as published by Lizzit et al. [11] consists of three components. We tried to confirm the existence of a third feature [12], but in principle it is not possible to achieve distinct findings during the fitting procedure. Due to intrinsic complexity of the B-peak, with the highest BE of the "true bulk" component, the obtained above BE difference, $0.360 \mathrm{eV}$, could be the first approximation of SCLS of the $\mathrm{Ru}(0001)$ surface. During adsorption of cobalt at $350 \mathrm{~K}$, the shape of the $\mathrm{Ru} 3 d_{5 / 2}$ peak changes. The intensity of the ruthenium surface peak, referred for convenience as $\mathrm{Ru}_{\mathrm{Ru}}^{\mathrm{V}}$ (top - vacuum, bottom ruthenium) decreases and the high BE region slightly increases in intensity. The case of 0.46 AML of Co is illustrated in the bottom inset of Fig. 2. The peak is decomposed into three contributions; i.e. the new interface component, I (referred as $\mathrm{Ru}_{\mathrm{Ru}}^{\mathrm{Co}}$ ), has appeared at $280.009 \mathrm{eV}$. The disappearance of surface $\mathrm{Ru}$ peak can be attributed to the removal of the vacuum-Ru interface at the expense of creation of a vacuum-Co and $\mathrm{Co}-\mathrm{Ru}$ interfaces.

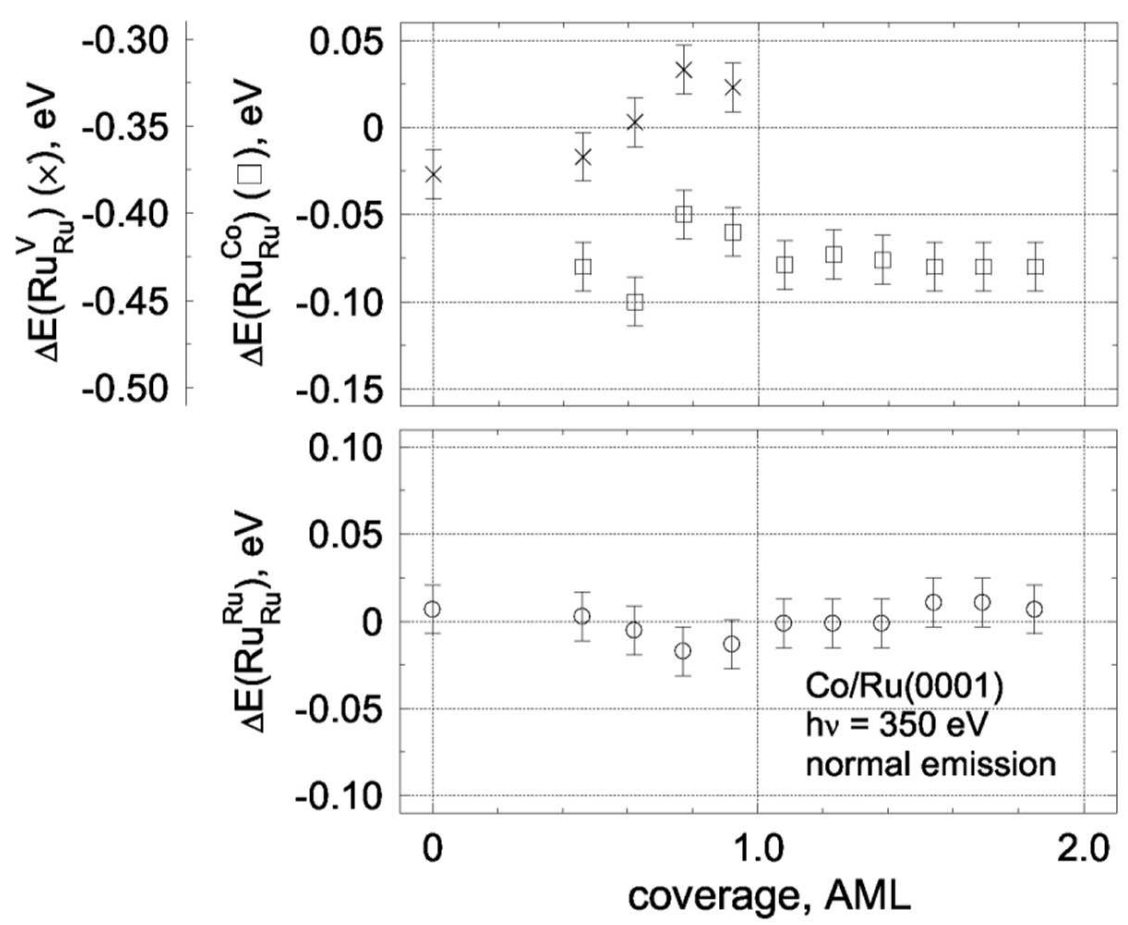

Fig. 3. Binding energy shifts of the three components of the Ru $3 d_{5 / 2}$ peak (SR-PS) as a function of cobalt coverage. Upper part: the surface $\Delta E\left(\mathrm{Ru}_{\mathrm{Ru}}^{\mathrm{V}}\right)$ (left scale, crosses) and the interface $\Delta E\left(\mathrm{Ru}_{\mathrm{Ru}}^{\mathrm{Co}}\right)$ (right scale, squares) contributions are given relative to the bulk component. Bottom part: the bulk $\Delta E\left(\mathrm{Ru}_{\mathrm{Ru}}^{\mathrm{Ru}}\right)$ peak relative to the average $(280.088 \mathrm{eV})$ value. 
The BEs shifts $(\Delta E)$ of the $\mathrm{Ru} 3 d_{5 / 2}$ components are collected in Fig. 3. A shift of the bulk contribution with cobalt coverage should be expected in the case of the alloying. However, from the figure (bottom part) negligible changes are observed suggesting that $\mathrm{Co}$ and $\mathrm{Ru}$ behave like independent metals. In the monolayer range, some perturbation of the surface and the interface line position with increasing amount of cobalt (upper graph: crosses and squares, respectively) are detected. The changes could be attributed to the short-range interaction between cobalt and ruthenium atoms in the upper layer. The BEs dependences indicate a very small electronic interaction between the two metals. For higher coverages, the interface component seems to be unchanged within experimental error.

\subsection{Valence band}

The valence band spectra of the clean $\mathrm{Ru}(0001)$ surface and the $\mathrm{Co} / \mathrm{Ru}(0001)$ interface obtained at $h \nu=40 \mathrm{eV}$ under normal emission are drawn in Fig. 4 . The spectra were registered in different experiments and reflect changes induced by cobalt at coverages of 0.49 AML (left part) and 0.69 AML (right part). In order to clearly identify changes, the difference spectra (bottom curves) are added to the graphs.

The VB of the clean ruthenium surface could be decomposed into four contributions as it was treated in published papers $[13,14]$. At the Fermi edge (BE = $0 \mathrm{eV}$ ), the intensity increases steeply ending with a soft shoulder of around $1 \mathrm{eV}$. Then, the main intensity of the band is positioned at $\mathrm{BE}=2.45 \mathrm{eV}$ and both features originate from the $\mathrm{Ru} d$-band of the top most and lower states, respectively. The second intense feature, observed at $5.68 \mathrm{eV}$, is attributed to a surface state. Finally, the weak emission at $7.8 \mathrm{eV}$ originates from a resonance state.

It is known that the VB spectra of bulk cobalt show $d$-band with the one asymmetric peak near $0 \mathrm{eV}$ of the width of $3.5 \mathrm{eV}$ which represents the maximum density of occupied state. The width of the $d$-band at the surface becomes much more narrow as compared with the bulk [15, 16] and for our coverages much more contracted feature should be expected. In fact, after adsorption of cobalt, the region near zero BE becomes much more steep whereas the energy position and the linewidth for every Ru-originating structure are the same (Fig. 4). The Ru-derived peaks (at 2.45, 5.68, and $7.80 \mathrm{eV}$ ) simply decrease in intensity. The difference curve shows a dominating contribution of the peak at $0.16 \mathrm{eV}$ below the Fermi level, which corresponds to the transition from the maximum density of states $(3 d)$ of a $2 \mathrm{D}$ single layer of cobalt. The separation between the $d$-bands of $\mathrm{Ru}$ and $\mathrm{Co}$ is equal to $2.29 \mathrm{eV}$ and due to this it seems that the interaction between $d$ orbitals in the Ru substrate and the Co overlayer should be rather weak. Finally, from careful inspection of the data it was concluded that the valence band spectra of the $\mathrm{Co} / \mathrm{Ru}(0001)$ interface could be composed of a linear combination of two separate components. 

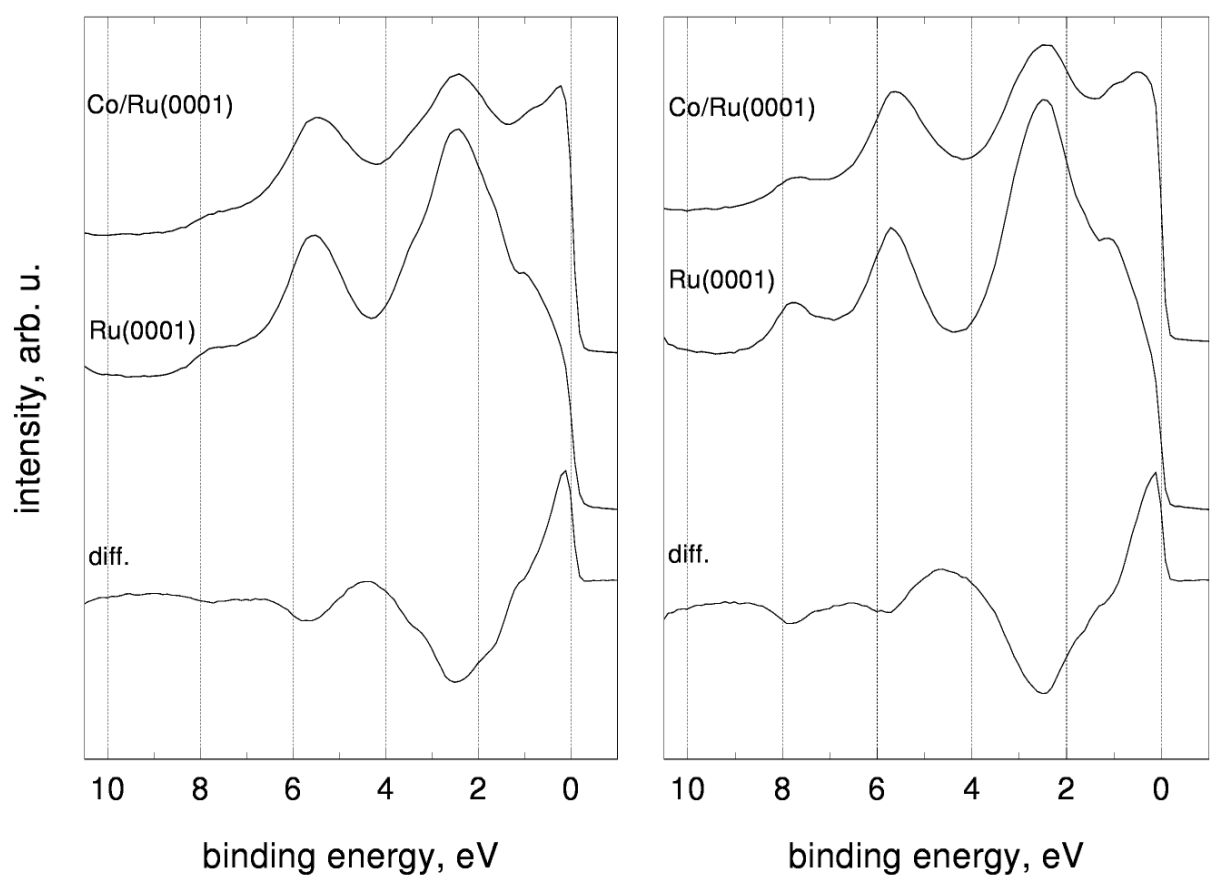

Fig. 4. Valence band photoemission spectra (SR-PS) recorded at indicated interfaces ( $h \nu=40 \mathrm{eV}$, normal emission). The cobalt coverage is equal to $0.49 \mathrm{AML}$ (left part) and 0.69 AML (right part). Bottom curves represent the difference spectra.

\section{Conclusions}

Deposition of cobalt on $\mathrm{Ru}(0001)$ at $350 \mathrm{~K}$ is going through a complete wetting of the first layer of adsorbate. The photoemission data do not show a large perturbation in the electronic properties of the metals. In the monolayer range, the $\mathrm{Co}-\mathrm{Co}$ distance follows the $\mathrm{Ru}-\mathrm{Ru}$ ones with a small net charge transfer from $\mathrm{Co}$ to $\mathrm{Ru}$. The $\mathrm{BE}$ changes with Co coverage are mainly due to the $\mathrm{Co}-\mathrm{Co}$ interactions at the new lattice geometry (pseudomorphism). Similarly, a change in the valence band is due almost exclusively to a geometrical effect.

\section{Acknowledgments}

This work was supported by the European Community - Research Infrastructure Action under the FP6 Structuring the European Research Area programme (through the Integrated Infrastructure Initiative Integrated Activity on Synchrotron and Free Electron Laser Science, contract number RII3-CT-2004-506008). One of us (J.O.) wants to acknowledge the support from the Danish National Scientific Research Council. 


\section{References}

[1] H.W. van Kesteren, F.J.A. den Broeder, P.J.H. Bloemen, E.A.M. van Alphen, W.J.M. de Jonge, J. Magn. Magn. Mater. 102, L9 (1992).

[2] R.Q. Hwang, C. Günther, J. Schröder, S. Günther, E. Kopatzki, R.J. Behm, J. Vac. Sci. Technol. A 10, 1970 (1992).

[3] C. Liu, S.D. Bader, J. Magn. Magn. Mater. 119, 81 (1993).

[4] http://www.webelements.com/.

[5] I. Daruka, A.-L. Barabási, Phys. Rev. Lett. 79, 3708 (1997).

[6] A.K. Schmid, J.C. Hamilton, N.C. Bartelt, R.Q. Hwang, Phys. Rev. Lett. 77, 2977 (1996).

[7] F. El Gabaly, S. Gallego, C. Muñoz, L. Szunyogh, P. Weinberger, C. Klein, A.K. Schmid, K.F. McCarty, J. de la Figuera, Phys. Rev. Lett. 96, 147202 (2006).

[8] T.H. Andersen, L. Bech, Z.S. Li, S.V. Hoffmann, J. Onsgaard, Surf. Sci. 559, 111 (2004).

[9] S. Valeri, A. Borghi, G.C. Gazzadi, A. di Bona, Surf. Sci. 423, 346 (1999).

[10] Landolt-Börnstein - Group III Condensed Matter, Vol. 24b, Springer-Verlag, Berlin 1994.

[11] S. Lizzit, A. Baraldi, A. Groso, K. Reuter, M.V. Ganduglia-Pirovano, C. Stampfl, M. Scheffler, M. Stichler, C. Keller, W. Wurth, D. Menzel, Phys. Rev. B 63, 205419 (2001).

[12] P.J. Godowski, J. Onsgaard, M. Trzebiatowska-Gusowska, K. Pater, Z.S. Li, Phys. Rev. B 73, 193403 (2006).

[13] M. Lindroos, P. Hofmann, D. Menzel, Phys. Rev. B 33, 6798 (1986).

[14] T. Pelzer, G. Ceballos, F. Zbikowski, B. Willerding, K. Wandelt, U. Thomann, Ch. Reuß, Th. Fauster, J. Braun, J. Phys., Condens. Matter 12, 2193 (2000).

[15] P. Heimann, E. Marschall, H. Neddermeyer, M. Pessa, H.F. Roloff, Phys. Rev. B 16, 2575 (1977).

[16] F.J. Himpsel, D.E. Eastman, Phys. Rev. B 21, 3207 (1980). 